\title{
Single-mode waveguides for GRAVITY
}

\section{The cryogenic 4-telescope integrated optics beam combiner}

\author{
K. Perraut ${ }^{1}$, L. Jocou ${ }^{1}$, J. P. Berger ${ }^{1}$, A. Chabli ${ }^{2}$, V. Cardin ${ }^{3}$, G. Chamiot-Maitral ${ }^{2}$, A. Delboulbé ${ }^{1}$, F. Eisenhauer ${ }^{4}$, \\ Y. Gambérini ${ }^{5}$, S. Gillessen ${ }^{4}$, S. Guieu ${ }^{1}$, J. Guerrero ${ }^{2}$, M. Haug ${ }^{4}, 6$, F. Hausmann ${ }^{4}$, F. Joulain ${ }^{3}$, P. Kervella ${ }^{7}$, \\ P. Labeye ${ }^{2}$, S. Lacour ${ }^{7}$, C. Lanthermann ${ }^{1}$, V. Lapras ${ }^{2}$, J. B. Le Bouquin ${ }^{1}$, M. Lippa ${ }^{4}$, Y. Magnard ${ }^{1}$, T. Moulin ${ }^{1}$, \\ P. Noël ${ }^{2}$, A. Nolot ${ }^{1}$, F. Patru ${ }^{1,7}$, G. Perrin ${ }^{7}$, O. Pfuhl ${ }^{4}$, S. Pocas ${ }^{2}$, S. Poulain ${ }^{3}$, C. Scibetta ${ }^{2}$, E. Stadler ${ }^{1}$, \\ R. Templier ${ }^{2}$, N. Ventura ${ }^{1}$, C. Vizioz ${ }^{2}$, A. Amorim ${ }^{8}$, W. Brandner ${ }^{9}$, and C. Straubmeier ${ }^{10}$
}

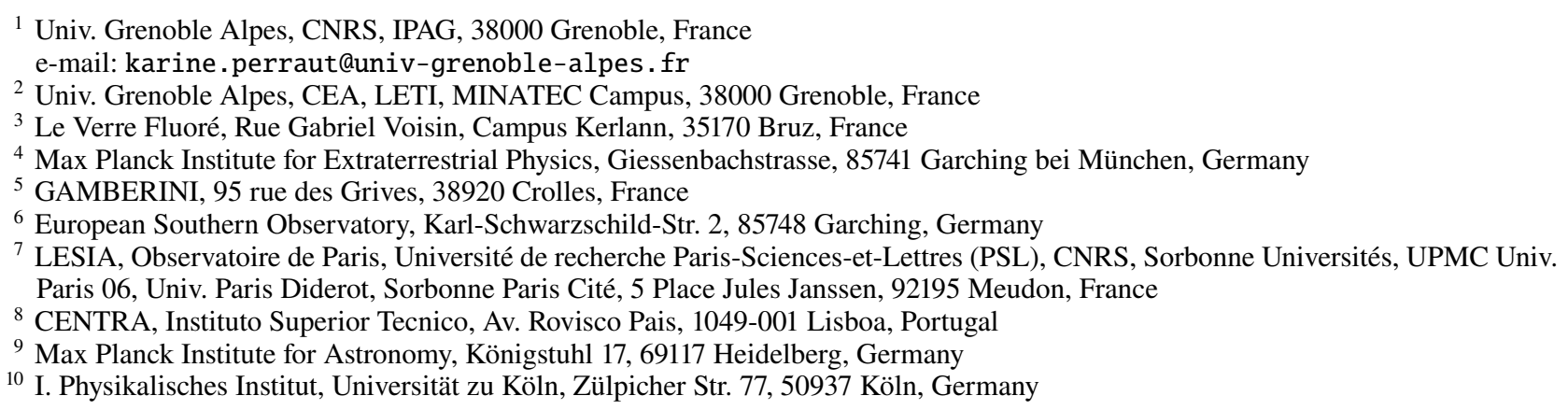

Received 23 December 2017 / Accepted 14 February 2018

\begin{abstract}
Context. Within the framework of the second-generation instrumentation of the Very Large Telescope Interferometer of the European Southern Observatory we have developed the four-telescope beam combiner in integrated optics.

Aims. We optimized the performance of such beam combiners, for the first time in the near-infrared $K$ band, for the GRAVITY instrument dedicated to the study of the close environment of the galactic centre black hole by precision narrow-angle astrometry and interferometric imaging.

Methods. We optimized the design of the integrated optics chip and the manufacturing technology as well, to fulfil the very demanding throughput specification. We also designed an integrated optics assembly able to operate at $200 \mathrm{~K}$ in the GRAVITY cryostat to reduce thermal emission.

Results. We manufactured about 50 beam combiners by silica-on-silicon etching technology. We glued the best combiners to singlemode fluoride fibre arrays that inject the VLTI light into the integrated optics beam combiners. The final integrated optics assemblies have been fully characterized in the laboratory and through on-site calibrations: their global throughput over the $K$ band is higher than $55 \%$ and the instrumental contrast reaches more than $95 \%$ in polarized light, which is well within the GRAVITY specifications.

Conclusions. While integrated optics technology is known to be mature enough to provide efficient and reliable beam combiners for astronomical interferometry in the $H$ band, we managed to successfully extend it to the longest wavelengths of the $K$ band and to manufacture the most complex integrated optics beam combiner in this specific spectral band.
\end{abstract}

Key words. techniques: high angular resolution - techniques: interferometric

\section{Introduction}

GRAVITY is the second-generation Very Large Telescope Interferometer (VLTI; Mérand et al. 2014) instrument for precision narrow-angle astrometry and interferometric imaging. Designed to probe physics close to the event horizon of the Galactic Centre (GC) black hole (Eisenhauer et al. 2011) but also to map the broad-line regions of active galactic nuclei or to image the close, complex, and variable environments of young stellar objects, it operates in the near-infrared $K$ band and benefits from the unique potential of the VLTI: four $8 \mathrm{~m}$ Unit Telescopes (UTs) for high angular resolution imaging at high sensitivity, and a 2 arcsec field that provides simultaneous interferometry of two objects and narrow-angle astrometry. As detailed in GRAVITY Collaboration (2017), the concept of GRAVITY follows the demanding requirements of these scientific cases and all the subsystems of the instrument have been designed and optimized to fulfil its high-level specifications: a $K$ band wavefront sensor that allows adaptive optics to be performed even in highly dust-extincted regions (Scheithauer et al. 2016; Deen et al. 2016), a fringe-tracker (Lacour, in prep.) equipped with a fast low-noise detector (Mehrgan et al. 2016) that is able to stabilize fringes on magnitudes better than $m_{\mathrm{K}} \sim 10$ for phase referencing in the GC field, and for long integration times on the 
scientific detectors up to $m_{\mathrm{K}} \sim 16$ to follow the black hole flares; a spectrometer with spectral resolutions of 22500 and 4500 (Straubmeier et al. 2014) that makes spectro-interferometry possible across near-infrared lines (e.g. hydrogen and helium) and $\mathrm{CO}$ bandheads; pupil and field control and stabilization (Anugu et al. 2018), and a metrology device (Lippa et al. 2016) that propagates all along the GRAVITY instrument up to the M2 mirrors of the telescopes for narrow angle astrometry with an accuracy of a few tens of microseconds of arc (Lacour et al. 2014) to probe the general relativistic effects around the GC.

At the heart of GRAVITY, there are beam combiners that combine four telescopes of the VLTI array, either the UTs or the $1.8 \mathrm{~m}$ Auxiliary Telescopes (ATs). With such imaging instruments in mind, Kern \& Malbet (1997) started a research and development programme in the 1990s to develop integrated optics (IO) chips for multi-telescope beam combinations (Malbet et al. 1999) with the aim of providing compact and stable beam combiners using single-mode guided optics for high-accuracy measurements (Coudé du Foresto et al. 1997). This research and technology programme was built on a combination of design and manufacturing, laboratory characterization, and on-sky demonstration of the most promising concepts. This led to the following demonstrations. Using the telecom developments made in the $1.3-1.7 \mu \mathrm{m}$ spectral range, several two-telescope combiner prototypes were manufactured with different planar optics technologies, such as ion exchange on glass (Benech 1997) or doped silica-on-silicon etching (Mottier 1997), and tested on sky in the 2000s. First, a two-telescope demonstrator in the $H$ band was installed on the IOTA interferometer (Berger et al. 2001), and then replaced by a three-telescope demonstrator (Berger et al. 2003) that was used for scientific observations until the IOTA array closed. These on-sky validations confirmed that the different IO manufacturing technologies were mature enough to provide reliable beam combiners for astronomical interferometry in the $H$ band, as clearly illustrated recently with the results obtained by the PIONIER instrument on the VLTI (Le Bouquin et al. 2011). On the contrary, for longer wavelengths like those covering the $K$ band, this was not the case. Extending the technologies already used in the telecom domains to the $K$ band was indeed a challenge mainly because of the material throughput. The first tests were done by Laurent et al. (2002) and Le Bouquin et al. (2006) with two-telescope beam combiners. While stability and high instrumental contrasts were pointed out, it was noted that throughput could be improved by optimizing the technological parameters, the beam combiner design, and the fibre/waveguide coupling.

Based on the successful demonstrations of the integrated optics technology for multi-telescope interferometric instrumentations, the GRAVITY consortium chose for its combination a concept based on an IO beam combiner to benefit from the alignment stability, the intrinsic modal filtering provided by the single-mode waveguides, and the compactness of the chip, which allows the implementation of the beam combiner in a cryostat. Given the stringent requirements of GRAVITY, mainly in terms of throughput, several developments and dedicated validations on several prototypes were mandatory.

In this paper, we report on these developments of the GRAVITY IO beam combiners and on their performance. We first recall the IO specifications derived from the GRAVITY high-level requirements (Sect. 2). Then we detail the design and the manufacturing of the integrated optics assembly (Sect. 3). We finally provide the performance we measured for each assembly in the lab and when installed at the heart of the GRAVITY instrument (Sect. 4.1).

\section{Specifications of the GRAVITY IO assembly}

These specifications were directly derived from the GRAVITY high-level requirements. We only detail in this section those that had a strong impact on the IO design and manufacturing.

\subsection{GRAVITY high-level requirements}

Combining four telescopes. The combination scheme chosen for GRAVITY is the outcome of a combination of laboratory and sky testing of different concepts and a system study like the one described in Le Bouquin et al. (2004). The beam combination concept of GRAVITY is based on a "pairwise static ABCD" scheme that allows us to extract four phase states of the coherent signal for each of the six baselines simultaneously, i.e. without temporal modulation.

Interferometric measurement accuracy. Reaching a $1 \%$ accuracy or better on the interferometric visibilities requires using single-mode waveguides over the operating spectral band, which constrains the radius of the mode that propagates along the waveguide or the numerical aperture of the waveguide.

Limiting magnitudes. As pointed out in the Introduction, one of the most demanding requirements is the throughput. Being able to fringe track on $m_{\mathrm{K}} \sim 10$ and to reach $m_{\mathrm{K}} \sim 16$ with long integrations requires optimizing the intrinsic transmission of each device and reducing the thermal background seen by the detectors. This has led to the implementation of the IO assemblies in the cryostat (Haug et al. 2012), which was a huge challenge since it was the first time that such IO beam combiners were used at cold temperatures.

Narrow-angle astrometry. To provide an accurate differential phase measurement to achieve astrometry with a few tens of micro-arcsecond accuracy, GRAVITY is equipped with a metrology device (Lippa et al. 2016): it includes a laser beam at $\lambda=1.908 \mu \mathrm{m}$ that is back-injected from the outputs of the beam combiners up to the secondary mirrors of the telescopes. As a consequence, the IO output should be anti-reflection coated at $1.908 \mu \mathrm{m}$ to avoid strong laser reflections inside the instrument towards the scientific detector.

\subsection{IO implementation and interfaces}

GRAVITY has two interferometric beam combiners: the first dedicated to the fringe tracking (FT) instrument that points to the phase reference star, the second for the science (SC) instrument that points to the scientific target. In both cases, the four input beams are coupled into single mode fibres ensuring the spatial filtering and the transport of the light up to the IO beam combiner. Thus, for the FT and the SC, the guided optics part is composed of five units of single-mode waveguides: (1) a fibre ensuring the collection of the VLTI flux, (2) a fibre section dedicated to differential optical path difference compensation between the fringe tracker and the science instrument (fibre differential delay line; FDDL), (3) a fibre polarization rotator (FPR), (4) a section gathering four fibres in a silicon array (hereafter called the $\mathrm{V}$-groove) that is connected to the integrated optics component (hereafter called the fibre array), and (5) an integrated optics beam combiner whose output acts as the entrance slit of the GRAVITY spectrograph (Fig. 1). Sections (2) and (3) compose the fibre control unit (FCU) which will be detailed in Paper II (Perrin et al. in prep.).

\subsection{IO specifications}

The high-level specifications of the IO assembly are summarized in the first two columns of Table 1 . The first set of specifications 


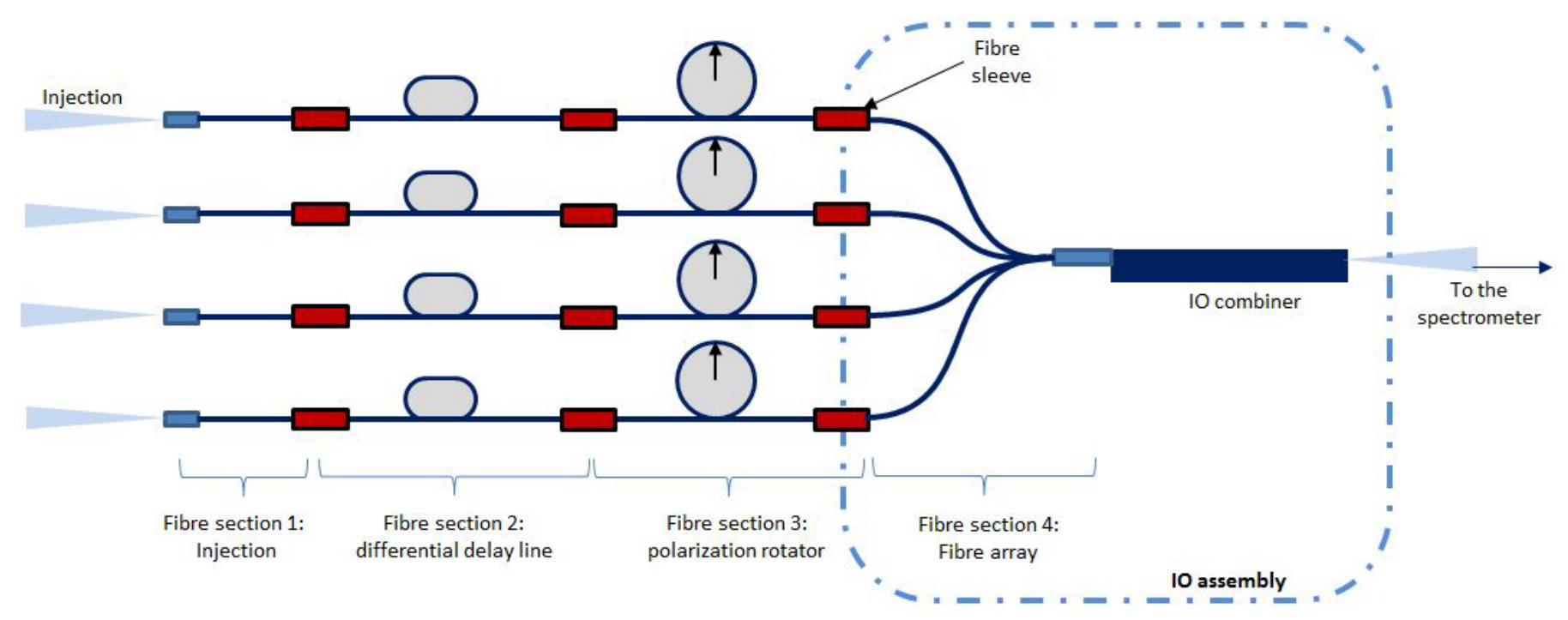

Fig. 1. Overview of the GRAVITY guided optics: fibre links and IO assembly (within the dash-dotted line) composed of a fibre array and an IO beam combiner, all of which implemented in the cryostat.

Table 1. High-level requirements and performance of the GRAVITY integrated optics beam combiners (lines 2-5) and of the IO assembly (lines 6-11).

\begin{tabular}{lccc}
\hline \hline Quantity & Requirement & Performance & Verification \\
\hline Input waveguide spacings & $1 \mathrm{~mm}-3 \mathrm{~mm}-1 \mathrm{~mm}$ & $1 \mathrm{~mm}-3 \mathrm{~mm}-1 \mathrm{~mm}$ & Design (Sect. 3.1) \\
Output waveguide spacing & $180 \mu \mathrm{m}$ & $180 \mu \mathrm{m}$ & Design (Sect. 3.1) \\
Phase shift between ABCD outputs & $90 \pm 10^{\circ a}$ & $85 \pm 3^{\circ}$ & Measurements (see Sect. 4.1) \\
Mode field radius & $3.8 \mu \mathrm{m}^{b}$ & $3.9 \pm 0.1 \mu \mathrm{m}$ & Measurements (see Sect. 4.2.1) \\
Operation wavelength range & {$[1.9 \mu \mathrm{m} ; 2.45 \mu \mathrm{m}]^{c}$} & {$[1.9 \mu \mathrm{m} ; 2.45 \mu \mathrm{m}]$} & Measurements (see Sect. 4.3.4) \\
Operation pressure & $10^{-6} \mathrm{mbar}$ & $10^{-6} \mathrm{mbar}$ & Design \\
Operation temperature & $200 \mathrm{~K}$ & $200 \mathrm{~K}$ & Design \\
Transmission (with connections) & $\geq 50 \%{ }^{a, d}$ & $\geq 55 \%$ & Measurements (see Sect. 4.2.1) \\
Instrumental contrast & $\geq 95 \% \%^{a, e}$ & $\geq 95 \%$ & Measurements (see Sect. 4.3.2) \\
Dispersion & $\leq 5 \mu \mathrm{rad} . \mathrm{cm}^{2 f}$ & $\leq 3 \mu \mathrm{rad} . \mathrm{cm}^{2}$ & Measurements \\
\hline
\end{tabular}

Notes. ${ }^{(a)}$ Over the $K$ band. ${ }^{(b)}$ At $\lambda=2.15 \mu \mathrm{m}$, this translates in a numerical aperture $(N A)$ which encircles $95 \%$ of the emitted flux of the Gaussian intensity distribution of $0.218 .{ }^{(c)} K$ band and metrology laser wavelength $(1.908 \mu \mathrm{m}) .{ }^{(d)}$ Goal: $\geq 55 \%$. ${ }^{(e)}$ In polarized light. ${ }^{(f)}$ For the entire fibre chain, including Fibre Control Unit. ${ }^{(g)}$ This value includes the Fibre Control Unit that is the main contributor to this quantity due to the longer fibre length (see Paper II for details).

concerns the beam combiner design itself as the phase shifts between the ABCD outputs and the mode field radius. This is common with the fibre specification since the coupling between the fibre and the waveguide is optimal only if their numerical apertures are equal. The spacing of the input waveguides is fixed by the V-groove characteristics, and the spacing of the output waveguides is defined so that the outputs are separated by an entire number of pixels when re-imaged onto the detector. The second set of specifications is related to the whole IO assembly or even to the whole guided optics chain as the dispersion (last line of the Table), which mostly concerns the fibre manufacturing (see Paper II for details).

With regard to the IO assembly, the most challenging specification is related to the operation temperature and derives from a trade-off between the thermal background seen by the detectors and the reliability and the ageing in the cold of the glues used for the assemblies (Sect. 3.3). Standard telecom glues are tested to a temperature of $-40{ }^{\circ} \mathrm{C}$ or $-55^{\circ} \mathrm{C}$, while a few glues have been tested to lower temperatures for very specific applications (e.g. military and outer space). A simulation of black-body emission has been performed for different temperatures of the beam combiner and its emissivity. We have considered a slit with an emissivity of 1 and whose dimensions are the same as those of the cold mask, i.e. $4.52 \mathrm{~mm}$ long and $220 \mu \mathrm{m}$ high (Sect. 3.4). Taking into account the background coming from this slit received by each pixel, we obtained Fig. 2 for the mediumresolution mode and an integration time of $30 \mathrm{~s}$. This background emission is compared to the SC detector readout noise. From this simulation, we fixed the operation temperature to $200 \mathrm{~K}$.

\section{Four-telescope integrated optics assembly}

As depicted in Fig. 3, each IO assembly is composed of an IO beam combiner, a fibre array, and the corresponding mechanical mount.

\subsection{Design and technology optimization of the IO beam combiner}

The circuit was designed by Labeye (2008) and is displayed at the bottom right panel of Fig. 3 . The couplers with a 66/33 theoretical coupling ratio (in red) and the Y-junctions (with a 50/50 


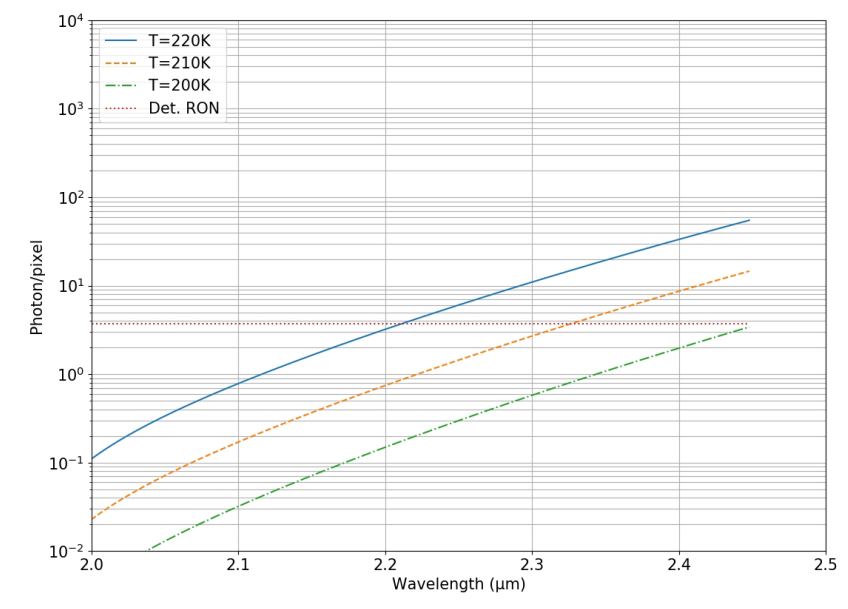

Fig. 2. Photon number recorded per pixel vs. wavelength for different temperatures of the SC IO beam combiner compared with the detector read-out noise (RON). The simulation is performed in medium spectral resolution for an integration time of $30 \mathrm{~s}$.

coupling ratio, in grey) respectively allow the light to be split into three beams of the same intensity and which feed the beam combination cells. As detailed in Benisty et al. (2009), this beam combination cell consists of a combination of two achromatic couplers (in green) and a $\pi / 2$-phase shifter (in light blue) that provide four outputs in quadrature directly sampling the coherent flux. The beam combiner has therefore 24 outputs ( 6 baselines $\times 4$ samples A,B,C,D) and for each baseline, four quadrature phase-states are simultaneously recorded.

We decided to use the silica-on-silicon etching technology based on doped silica deposited by plasma-enhanced chemical vapor deposition (PECVD) based on our know-how in the $H$ band with this technology mastered by the CEA/LETI. We had to optimize it for the $K$ band, mainly in terms of throughput. This was challenging since in the $K$ band we had to cope with the lower transmission of the silica and with $\mathrm{OH}$ absorption bands, especially around $2.2 \mu \mathrm{m}$. Extrapolating throughput measurements on a $80 \mathrm{~mm}$ combiner optimized for the $H$ band from Benisty et al. (2009) we decided that a specific effort should be spent to shorten the component length.

With respect to the design described in Benisty et al. (2009), we thus decided to optimize the optical function design by replacing the tricouplers to split the light with couplers and Yjunctions and by reducing the length of the phase-shifters that were no longer perfectly achromatic. Then we increased the index difference between the core and the outer layers to better confine the light, to be able to use stronger curvatures of the waveguides, and to reduce the length of the beam combiner and thus the absorption losses. To control as best we could the waveguide index difference we had to carry out systematic measurements of the index difference dependence to the level of doping. This was done through the use of the M-lines technique (Monneret et al. 2000). We then chose the doping level of the different layers to reach an index difference of about 0.016 . Finally, we fixed the waveguide sizes to ensure that the numerical aperture and the coupler balance are within the specifications over the $K$ band. We manufactured square waveguides with a width and a height of $5.8 \mu \mathrm{m}$. These values were chosen to have a cut-off wavelength of $1.85 \mu \mathrm{m}$. This leads to a mode field radius of $\omega_{0}$ of $3.9 \mu \mathrm{m}$. All these improvements led to a fourtelescope beam combiner on a $50 \mathrm{~mm}$ chip (Fig. 3, bottom left). Several chips with slightly different index differences around
0.016 were included in a $200 \mathrm{~mm}$ mask for the manufacturing by photolithography.

With regard to birefringence in the waveguides, the contributions can be divided in two categories: structural birefringence and stress-induced birefringence. Considering the index contrast and the geometry, the structural birefringence is well below the accuracy of any mode solver available. By introducing a small asymmetry of $0.1 \mu \mathrm{m}$ between the width and the height of the waveguide, we obtained a structural birefringence of $2 \times 10^{-6}$, leading to a beat length between the two polarizations of $55 \mathrm{~cm}$ at the central wavelength of the $K$ band. The birefringence induced by stress is far more difficult to evaluate. When it is possible to measure a stress in a thin layer deposited on a substrate, the stress in rectangular waveguides tends to relax after etching. We have not made any particular studies on $K$ band waveguides, but it has been established (on telecom components; private communication) that silica channel waveguides manufactured by this technology have a birefringence of around $10^{-5}$ leading to a beat length above $10 \mathrm{~cm}$ at the central wavelength of the $K$ band.

\subsection{Manufacturing of the 10 beam combiner}

The IO beam combiner is a silicon chip with three different silica layers deposited on a standard grade $200 \mathrm{~mm}$ silicon mechanical substrate. The first silica layer is called the "optical substrate" and is made of the purest possible silica. It was obtained by a wet thermal oxidation of the silicon substrate and was realized by the Vegatech company specialized in thermal oxidation. The second silica layer is called the "core layer" and was obtained by PECVD in an Applied Materials Centura $5200 \mathrm{E}$ apparatus. It was obtained by decomposition and oxidation of silane and was slightly doped with germanium dioxide $\left(\mathrm{GeO}_{2}\right)$ to obtain a refractive index difference of 0.016 between the core layer and the optical substrate. It was followed by a three-hour annealing at $1000{ }^{\circ} \mathrm{C}$ in a Vatran VT300 oven to remove the $\mathrm{OH}$ bonds present in the layer. The waveguides were then defined by photolithography and etching using an ASML ASM300 stepper (whose working wavelength is $248 \mathrm{~nm}$ ) and a custom modified EMAX reactive ion etching chamber using standard trifluoromethane chemistry. We specifically developed a stitching technique on our ASM300 stepper to manufacture the $20 \mathrm{~mm} \times 50 \mathrm{~mm}$ IO beam combiner using three repeating fields of $20 \mathrm{~mm} \times 16.6 \mathrm{~mm}$. Stitching accuracy was measured thanks to electronic microscope measurements; we obtained differences between two successive parts that were smaller than $50 \mathrm{~nm}$ and a mean value of $25 \mathrm{~nm}$, which is fully acceptable for $5.8 \mu \mathrm{m}$ square waveguides. Finally, the waveguides were covered with the third silica layer called the "superstrate layer" that was obtained by three successive PECVD TEOS (tetraethyl orthosilicate, formally named tetraethoxysilane) depositions of silica in the second chamber of our Applied Materials Centura 5200E apparatus followed by three annealing cycles at $1000^{\circ} \mathrm{C}$ (lasting three hours each) in the VT300. Silica layers were also deposited on the back side of the wafers in order to compensate for the deformation of the silicon wafers due to thermal stress induced by the mismatched thermal expansion coefficient of silica and silicon. It is indeed mandatory to keep the deformation bow of the silicon wafers below $100 \mu \mathrm{m}$ at each step of the process to be compatible with $200 \mathrm{~mm}$ silicon wafer fabrication equipment (especially photolithography).

Once diced and polished, each combiner was checked in terms of throughput, numerical aperture, and interferometric behaviour (Sect. 4.1). For the best IO beam combiners, the output face is coated by the Laser Zentrum company with an 


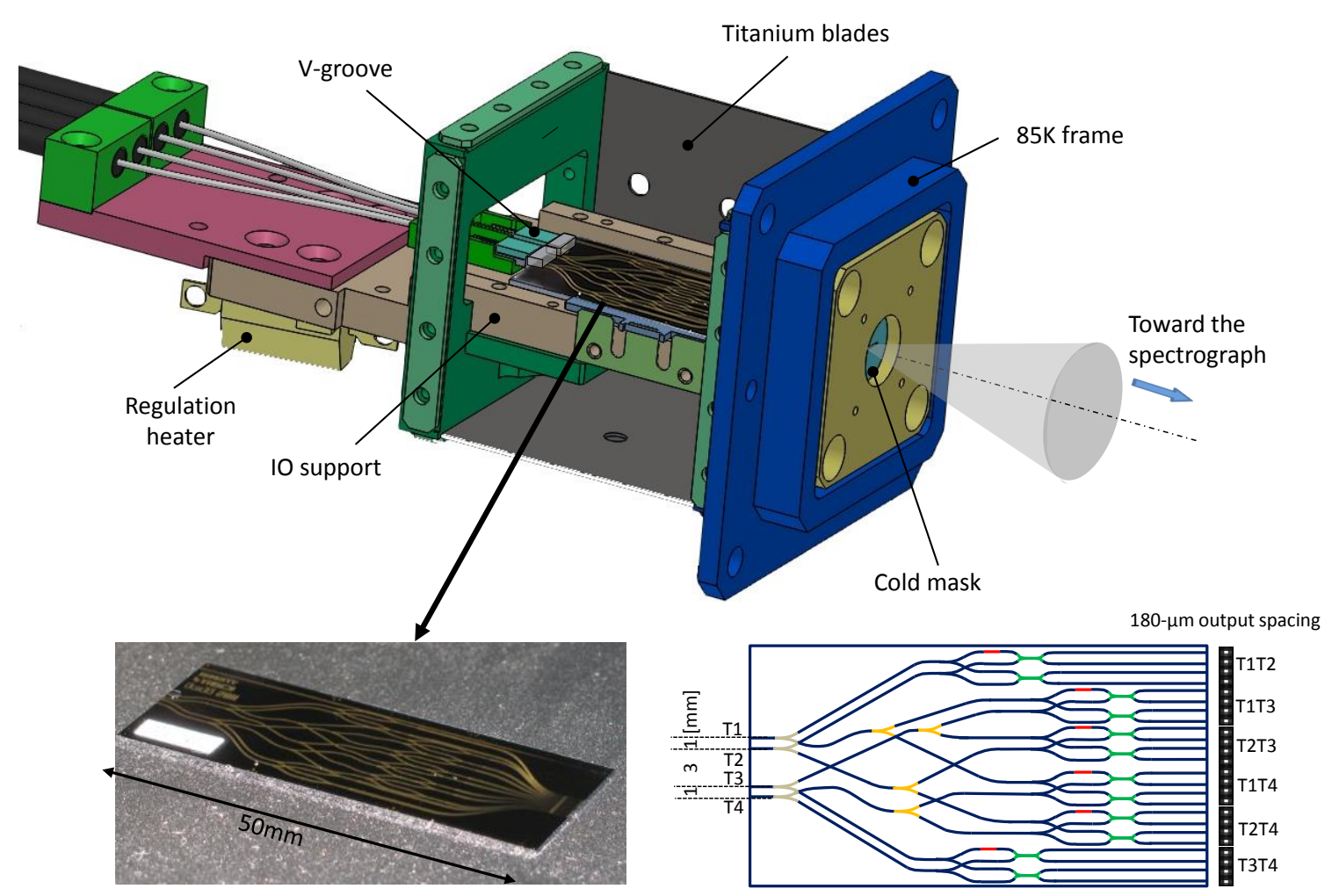

Fig. 3. Description of the IO assembly. Top: mechanical mount including the IO beam combiner and the fibre array. Bottom: photograph of an IO beam combiner manufactured by silica-on-silicon etching (left) and scheme of the beam combiner principle (right): 66/33 couplers (in red), Y-junctions (in grey), achromatic $\pi / 2$-phase shifters (in light blue), and 50/50 couplers (in green). See text for details.

Table 2. General characteristics of the fluoride fibres manufactured by Le Verre Fluoré.

\begin{tabular}{lc}
\hline \hline Core diameter & $6.5 \mu \mathrm{m}$ \\
Cladding diameter & $125 \mu \mathrm{m}$ \\
Cut-off wavelength & $1.88 \mu \mathrm{m}$ \\
Mode field radius (at $\lambda=2.15 \mu \mathrm{m})$ & $3.78 \mu \mathrm{m}$ \\
Beat length & $>200 \mathrm{~m}$ \\
\hline
\end{tabular}

anti-reflection coating which transmits the whole $K$ band and limits the reflection of the metrology laser at $1.908 \mu \mathrm{m}$.

\subsection{Manufacturing of the fibre array}

We used ZBLAN ${ }^{1}$ fibres manufactured by Le Verre Fluoré that ensure the best throughput in the $K$ band. The general characteristics of these fluoride glass fibres are given in Table 2. The fibres were specified with the lowest possible birefringence to be as neutral as possible in terms of polarization (see Paper II for details). The fibre array manufactured by Le Verre Fluore is connected to the FPR with E2000 connectors that ensure a low coupling loss (typically $0.1 \mathrm{~dB} /$ connection) and aims at arranging the incoming fibres so as to be connected to the IO beam combiner (Fig. 3, top). Thus, at the output end, the fibres have to present the same spacing as the IO input waveguides, e.g. 1,3 , and $1 \mathrm{~mm}$. This is performed thanks to two independent V-grooves supporting the fibres being directly glued on the IO chip and providing enough degrees of freedom to adjust the fine alignment between the fibre cores and the waveguides (Fig. 4).

\footnotetext{
1 Fluoride glass with a composition $\mathrm{ZrF} 4-\mathrm{BaF}_{2}-\mathrm{LaF}_{3}-\mathrm{AlF}_{3}-\mathrm{NaF}$
}

Each fibre array consists of several pieces of different materials that are glued together; the main challenge in the manufacturing process was to select the relevant glues for each part to compensate as much as possible for the dilatation coefficient gap, and thus limit constraints applied on each piece when cooling down to $200 \mathrm{~K}$. We emphasize that it is the first time that fibre devices of this kind have operated at such low temperatures. To select the glues, we decided to test different glues used at low temperatures for spatial instruments and developed several prototypes to be thermally cycled with a dedicated dewar.

Each V-groove consists of a silicon fibre holder glued to a titanium short piece. For this step we rejected the 353ND glue which turned out to be too hard and thus led to V-groove breakage. We tested three different soft glues (2216-3M, 302-3M, and OG-142-87) and finally selected 2216-3M which leads to undamaged V-grooves after 20 thermal cycles between $-90^{\circ}$ and $+30^{\circ}$. We mastered the quantity, and thus the thickness, of the glue deposited by using nylon wires as shims between the silicon holder and the titanium piece. On the silicon holder side, two fibres spaced by $1 \mathrm{~mm}$ were glued with the 2216-3M glue and covered with a glass lid. At the output of the silicon holder the fibres were left free to enter a hytrel cable that protects the fibres up to the E2000 connectors. Finally, in order to protect the fibres between the glass lid and the hytrel entrance, a soft epoxy resin (EPOTEK 310M) was deposited to reduce the constraints on the fragile fluoride fibres. Before this last operation, a drop of UV DESOLITE 3471 was deposited to seal the entrance of the hytrel cable to thus prevent the $310 \mathrm{M}$ from migrating inside. After all the gluing steps, the front end of the V-groove was polished to ensure a high optical quality since this part was to be glued to the IO beam combiner. 


\section{Fibre array front view}
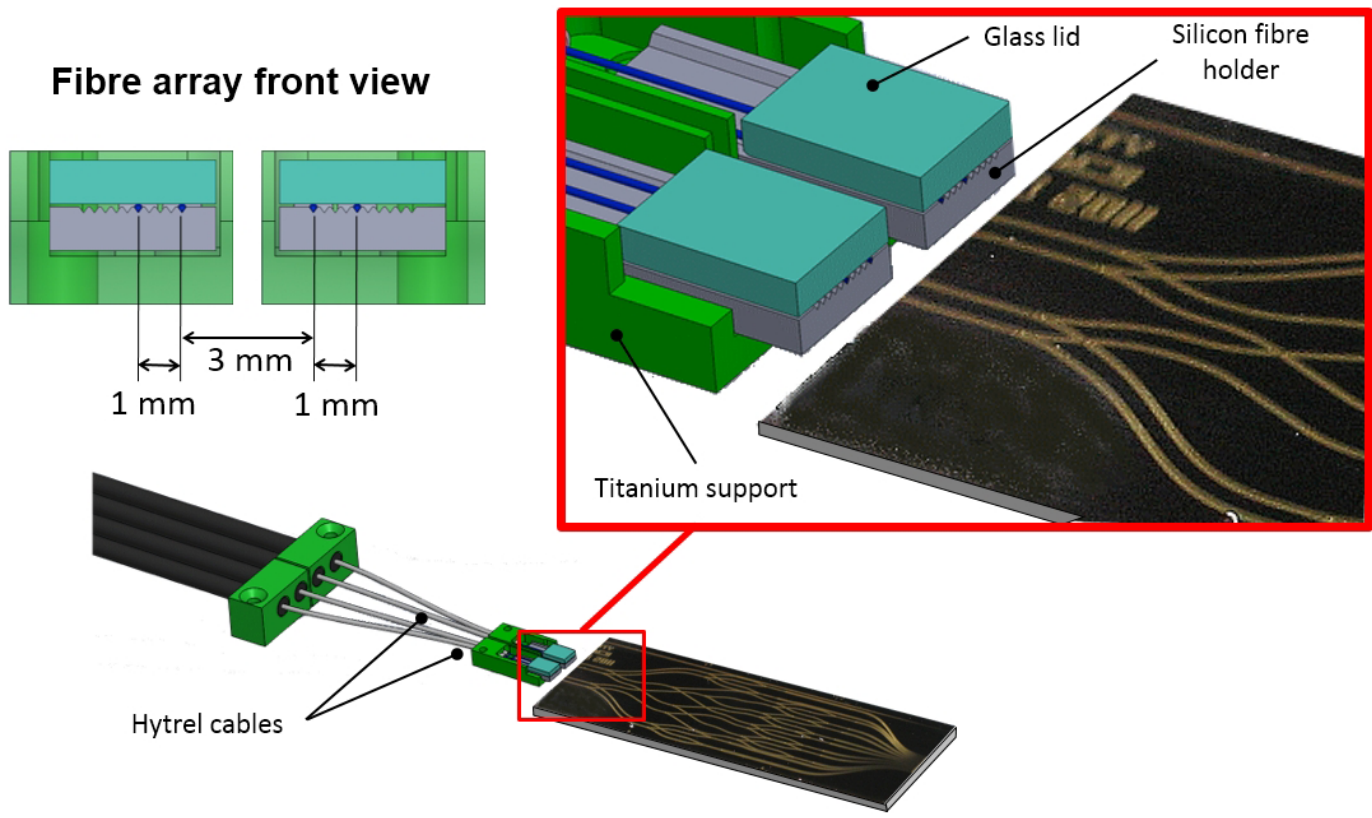

Fig. 4. Description of the fibre array. See text for details.

For each IO assembly, two V-grooves were manufactured and then glued to an IO beam combiner with the UV EPOTEK 142-87 glue (Fig. 4). Each V-groove fed the closest input waveguides (T1-T2 and T3-T4, respectively; Fig. 3, bottom right). The choice of feeding the IO beam combiner with two V-grooves allowed the fibres to be accurately aligned in front of the corresponding input waveguides to optimize the fibre/waveguide coupling. This also allowed us to cope with the fact that the core of the fluoride fibre could be decentred (by less than $1 \mu \mathrm{m}$ ) with respect to the cladding, which prevented the distance between the cores of two successive fibres in a V-groove from equaling the distance between the two grooves. During the gluing process, we fed the V-groove fibres with a source and measured the flux at different outputs of the IO beam combiners to check the matching between the fibre core and IO waveguides. If the matching is not perfect, a trade-off is made between the two fibres of a $\mathrm{V}$-groove, and photon losses are measured for the throughput budget (see Sect. 4.2.1). When the matching is maximized, the glue is UV polymerized to "freeze" the adjustment.

\subsection{Mechanical IO mount and thermal control}

The IO mount was designed to ensure an accurate positioning of the IO assembly with respect to the spectrometer and to allow thermal control of the IO assembly.

Since the beam combiner output acts as the spectrometer entrance slit, the mechanical mount concept should ensure a positioning of the IO outputs with respect to the detectors at a $10 \mu \mathrm{m}$ level. This was guaranteed by reference pins at the spectrometer front end (Fig. 5) and by an $X$ and $Y$ alignment performed in the lab with a collimating telescope. With regard to the focus adjustment (i.e. along the $Z$-axis), a preliminary adjustment was performed in the lab, taking into account the contraction in the cold of the different mechanical pieces thanks to a thermal study of the whole mount. This focus can be finely adjusted in operation thanks to the spectrometer motors.

Since it operates at $200 \mathrm{~K}$, while the spectrometer is cooled to $85 \mathrm{~K}$, the IO assembly was mounted to the $85 \mathrm{~K}$ frame through a
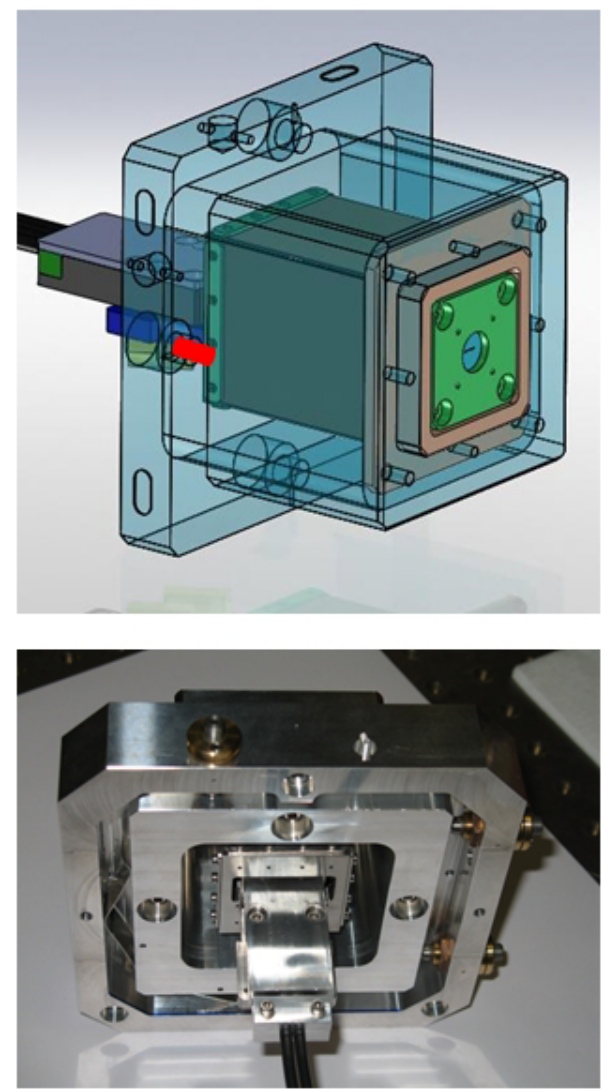

Fig. 5. Top: front end of the IO mount with one reference pin (in red) to be fixed on the spectrometer front end. Bottom: photo of the IO mount back end with the IO assembly screwed into it.

set of four $0.5 \mathrm{~mm}$ thick titanium blades that ensure both the stiffness of the whole assembly and the thermal insulation between the IO beam combiner and the spectrometer (Fig. 3, top; for clarity only two blades are shown). The insulation was simulated for different blade materials and thicknesses. In addition, a heater was screwed below the IO assembly so as to bring a heat flow 

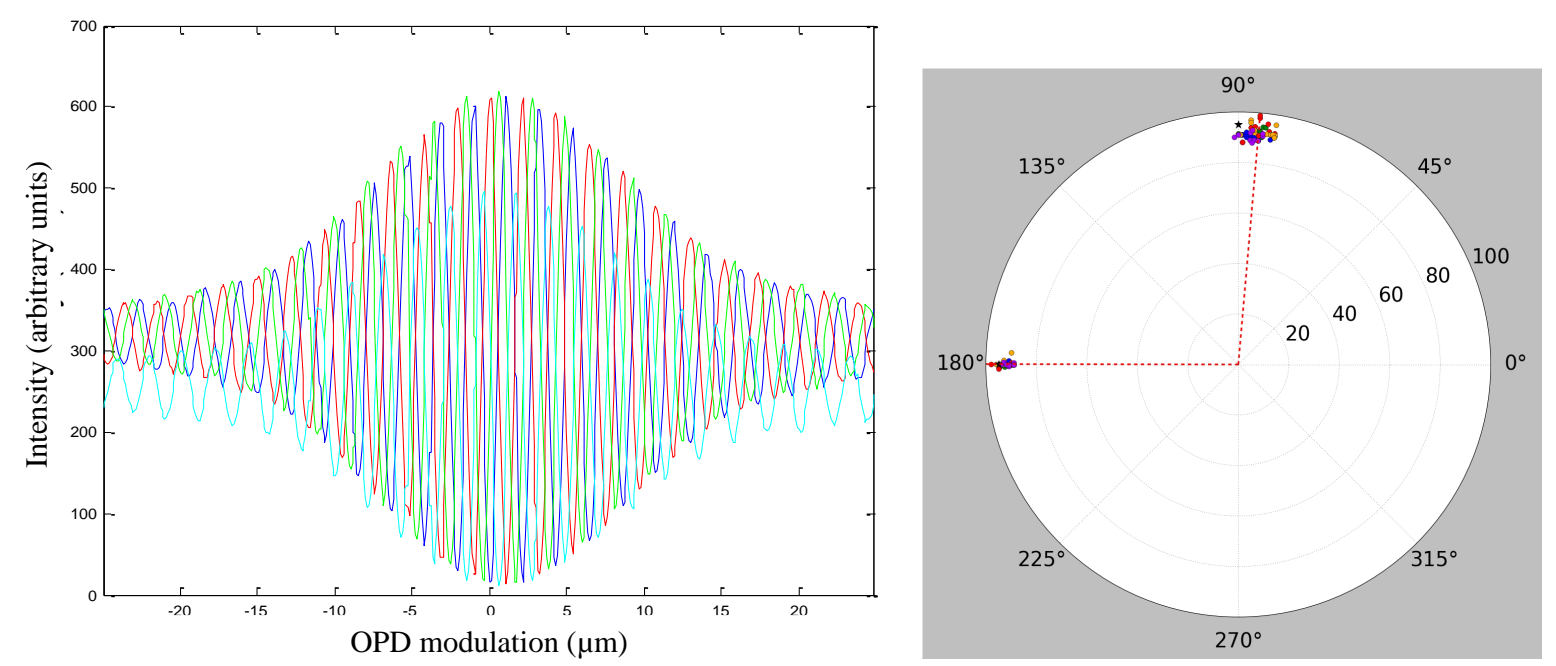

Fig. 6. Left: typical lab fringes obtained on one baseline when scanning the optical path difference (OPD). The four fringe patterns are in quadrature. Right: polar diagram of the 120 contrasts (along the radius) and 120 phase shifts (along the perimeter) measured in the lab over the $K$ band for the IO assemblies. Each colour corresponds to an assembly. The dotted lines display the mean phase shift values. For comparison with the requirements, we displayed the phase differences designed to be $90^{\circ}$ or $180^{\circ}$.

of $0.95 \mathrm{~W}$ required to thermally regulated the assembly around $200 \mathrm{~K}$. This heater is servoed by a LakeShore controller, which allows the IO assembly temperature to be adjusted in operation if needed. Finally, to reduce the thermal background seen by the detectors as much as possible, a cold mask was mounted on the " $85 \mathrm{~K}$ frame" (see Fig. 3, top). This mask is a slit that is $4.52 \mathrm{~mm}$ long and $220 \mu \mathrm{m}$ high. It was set about $200 \mu \mathrm{m}$ in front of the IO output. Its fine alignment with respect to the IO beam combiner output was performed in the lab with the same collimating telescope used for the IO mount alignment.

\subsection{Manufacturing of 10 assemblies}

For the GRAVITY project, we manufactured five IO assemblies. One was installed in the fringe tracker, another in the scientific instrument, and the others are spare parts. We characterized these five assemblies in the lab, and we present their performance in the next section.

\section{Results and performance}

Since the IO assemblies are at the heart of the GRAVITY instrument, some specifications can be checked in the lab at the IO assembly level, but some others can only be checked on-site inside the whole instrument.

\subsection{Validation of the $1 O$ beam combiners}

Mode field radius. We measured the numerical aperture by farfield imaging for all the beam combiners and we obtained a mean numerical aperture of $N A=0.20 \pm 0.02$, which fits the specification of the mode field radius.

Phase shifts between the ABCD outputs. With a dedicated interferometric testbench which is fully described in Benisty et al. (2009), we recorded lab fringes (Fig. 6, left) and measured the phase shifts over the $K$ band of all the beam combination cells of the five beam combiners of the IO assemblies, leading to 120 measurements. The average $\pi / 2$-phase shift provided by the phase-shifters is $85 \pm 3^{\circ}$ while the average $\pi$-phase shift that is ensured by the coupler design is $179.9 \pm 0.5^{\circ}$ (Fig. 6, right), which is in full agreement with the specifications.
Table 3. Typical throughput measurements on an integrated optics assembly.

\begin{tabular}{ccccc}
\hline \hline$\lambda[\mu \mathrm{m}]$ & 2.0 & 2.2 & 2.37 & $\mathrm{rms}$ \\
\hline Fibre array & & & & \\
Fibre + connectors & 0.96 & 0.96 & 0.96 & 0.01 \\
Fitting mode sizes & 0.985 & 0.985 & 0.985 & 0.005 \\
Glue & 0.99 & 0.99 & 0.95 & 0.02 \\
Matching pitchs & 0.97 & 0.97 & 0.97 & 0.01 \\
Coupling at 200 K & 0.95 & 0.95 & 0.95 & 0.02 \\
\hline Integrated Optics chip & & & & \\
IO chip & 0.87 & 0.60 & 0.58 & 0.02 \\
Anti-reflection coating & 0.99 & 0.99 & 0.99 & 0.005 \\
\hline Total & 0.74 & 0.51 & 0.48 & 0.05 \\
Mean throughput & & 0.60 & & 0.05 \\
\hline
\end{tabular}

Notes. The values are similar for both polarizations.

\subsection{Validation of the $1 O$ assemblies}

\subsubsection{Throughput budget}

As pointed out in the Introduction, one of the most demanding specifications is the throughput. We present in this section the throughput budget on the IO assembly for three different wavelengths. The different contributions to the global throughput are as follows (see also Table 3):

- The fibre throughput: this value was measured for all the fibres by Le Verre Fluoré company at $2.2 \mu \mathrm{m}$.

- The fitting of IO and fibre mode size: the photon losses due to the mismatch between the mode sizes were computed from an overlap integral. The IO mode size was deduced from the numerical aperture measurement, while the fibre mode size was determined by simulations by Le Verre Fluoré and checked by numerical aperture measurements.

- The throughput of the glue: we estimated the throughput for a thickness of $5 \mu \mathrm{m}$ by using the throughput curve provided by the manufacturer (EPOTEK 142-87), and we checked that this estimation was fully consistent with our measurements. 


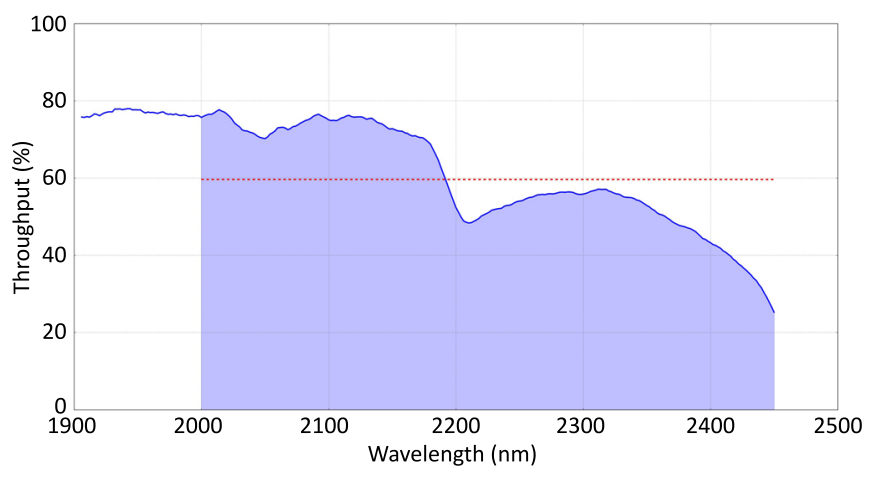

Fig. 7. Throughput over the $K$ band of an IO assembly. The absorption of the $\mathrm{OH}$ band in the silica can be seen around $2.2 \mu \mathrm{m}$. The red dashed line shows the mean throughput over the $2.0-2.45 \mu \mathrm{m}$ range. See text for details.

- The matching between the V-groove and IO pitches: these losses are due to the mismatch of the pitch of the fibre $\mathrm{V}$-groove with respect to the pitch of the IO input waveguides. These photon losses were measured during the gluing process (see Sect. 3.3).

- The operation at low temperature: to determine these losses, each assembly was cooled down to $190 \mathrm{~K}$ by means of a cryo-chamber and the flux variation versus temperature was measured during several thermal cycles. In practice, the losses were low for all assemblies and we adopted a conservative value of $5 \%$ as the flux losses in the cold (i.e. a coupling at $200 \mathrm{~K}$ of 0.95 ).

- The throughput of the IO chip: for each IO beam combiner, the throughputs at $2.0 \mu \mathrm{m}, 2.2 \mu \mathrm{m}$, and $2.37 \mu \mathrm{m}$ were measured as well as the spectral answer of a straight waveguide by means of a spectrometer. The spectral answer of each beam combiner was computed from that of the straight waveguide by taking into account the function losses. Then we checked that the computed value at $2.2 \mu \mathrm{m}$ was consistent with the measured value on our test bench, else we scaled the spectral answer to fit in. It should be noted that our optimization of the annealing temperature has drastically reduced the $\mathrm{OH}$ absorption around $2.2 \mu \mathrm{m}$; the throughput decrease at $2.2 \mu \mathrm{m}$ is 1.5 times less deep than that measured on the initial waveguides.

- The throughput of the anti-reflection coating: this throughput has been measured by the manufacturer. The mean throughput over the $K$ band is $99 \%$, while the reflection coefficient at $1.908 \mu \mathrm{m}$ is $0.06 \%$.

Finally we checked the global throughput at $2.2 \mu \mathrm{m}$ with a dedicated testbench. All the manufactured assemblies had an average throughput over the $K$ band higher than $50 \%$ (Fig. 7), which is within the GRAVITY specifications.

\subsubsection{Interferometric quantities}

We used our interferometric testbench to measure the instrumental contrasts of the five IO assemblies, which corresponds to 120 measurements. From a statistical approach, we derived an average contrast of $92 \pm 2 \%$ over the $K$ band (Fig. 6). However, we note that this average contrast corresponds to a rough estimation of the instrumental contrast since neither the birefringence nor the dispersion could be perfectly compensated within our testbench contrary to what is done in the GRAVITY instrument. Moreover our data reduction pipeline was not as accurate as the GRAVITY one and we estimated that our contrast measurement could be biased at a 5\% level. This validation was

\begin{tabular}{|c|c|c|c|c|}
\cline { 2 - 5 } \multicolumn{1}{c|}{} & TEL 1 & TEL 2 & TEL 3 & TEL 4 \\
\hline $1-2$ & 50.4 & 49.4 & 0.1 & 0.1 \\
\hline $1-3$ & 50.1 & 0.3 & 49.5 & 0.2 \\
\hline $1-4$ & 49.9 & 0.3 & 0.1 & 49.7 \\
\hline $2-3$ & 0.1 & 50.3 & 49.4 & 0.1 \\
\hline $2-4$ & 0.2 & 50.0 & 0.1 & 49.7 \\
\hline $3-4$ & 0.1 & 0.2 & 50.3 & 49.4 \\
\hline
\end{tabular}

Fig. 8. Example of the FT $\kappa$-matrix. See text for details.

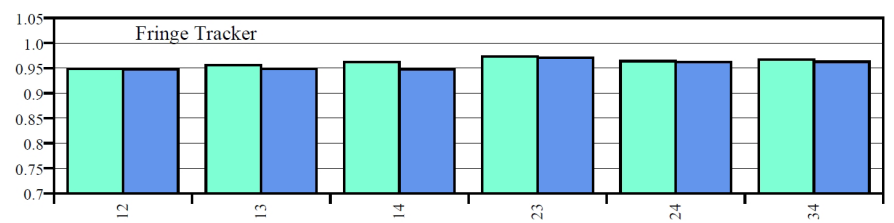

\begin{tabular}{|c|c|c|c|c|c|c|}
\cline { 2 - 7 } \multicolumn{1}{c|}{} & $1-2$ & $1-3$ & $1-4$ & $2-3$ & $2-4$ & $3-4$ \\
\hline $1-2$ & 99.3 & 0.1 & 0.2 & 0.1 & 0.2 & 0.0 \\
\hline $1-3$ & 0.1 & 99.6 & 0.1 & 0.1 & 0.0 & 0.1 \\
\hline $1-4$ & 0.1 & 0.1 & 99.4 & 0.0 & 0.2 & 0.1 \\
\hline $2-3$ & 0.2 & 0.2 & 0.0 & 99.4 & 0.1 & 0.1 \\
\hline $2-4$ & 0.1 & 0.0 & 0.1 & 0.1 & 99.7 & 0.1 \\
\hline $3-4$ & 0.0 & 0.2 & 0.2 & 0.3 & 0.3 & 99.1 \\
\hline
\end{tabular}

Fig. 9. Top: example of the FT instrumental contrast for the two polarizations (in different colours) and all the baselines. Bottom: wavelength averaged coherent flux transmission expressed as a percentage of the total transmitted coherent flux per baseline.

just a way to check that all beam combiners behaved correctly. The fine qualification of the instrument contrast could only be performed inside GRAVITY in operation (Sect. 4.3).

\subsection{On-site calibrations}

The data reduction of GRAVITY (Lapeyrere et al. 2014) is based on the principle of the pixel-to-visibility matrix (P2VM; Tatulli et al. 2007), which characterizes the relations of the photometry, the coherence, and the phase between the 4 inputs and the 24 outputs of the IO beam-combiner. These P2VMs are part of the instrument calibration and are performed for each observing mode at the end of each night of observations. As a byproduct, they provide fruitful information about the IO assembly behaviour and are a very useful diagnosis tool of instrument health.

\subsubsection{Photometric throughput}

The first information provided by the P2VM is the flux contribution of each beam to each fringe pattern, which provides the so-called $\kappa$-matrix. Ideally, for each baseline $i-j$, each input beam (i.e. TEL $i$ and TEL $j$ ) should contribute half of the flux to the fringe pattern, while the others contribute with a null flux, else cross-talk is observed. We have checked that the cross-talk is below $0.3 \%$ and that on average, each telescope contributes to each corresponding fringe pattern with a flux of $49.8 \pm 0.4 \%$ (Fig. 8).

\subsubsection{Instrumental contrast and coherence throughput}

The P2VMs also allow us to extract the instrumental contrast and the wavelength averaged coherent flux for each beam combiner. We have checked that for both polarizations the instrumental contrast reached $95 \%$ in polarized light, which is in full agreement with the instrumental contrast specification (Fig. 9, top). When the coherent flux is expressed as a percentage of the total 

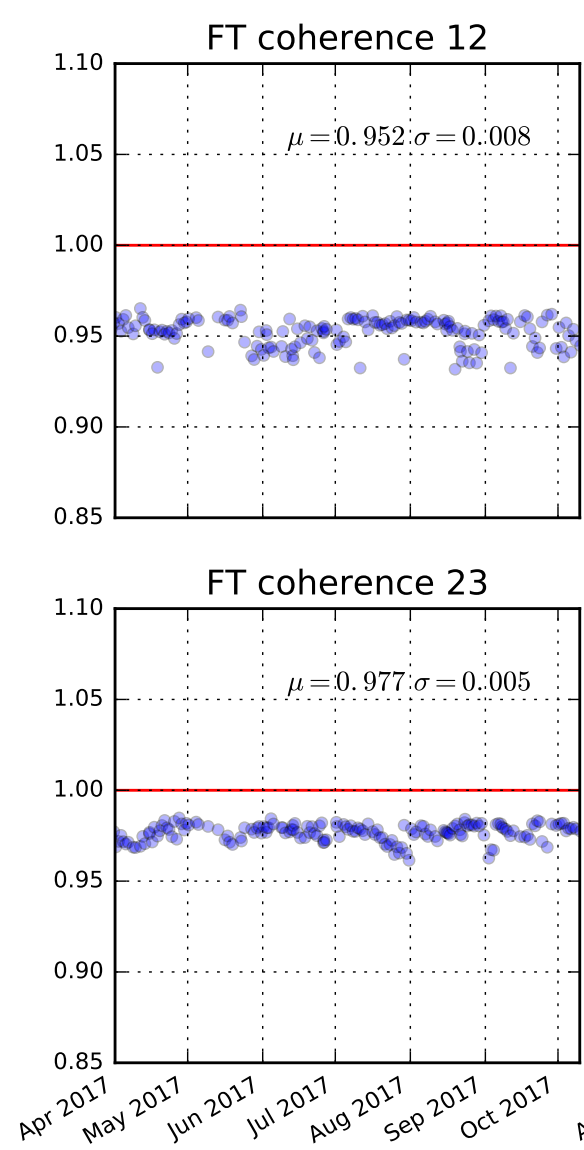

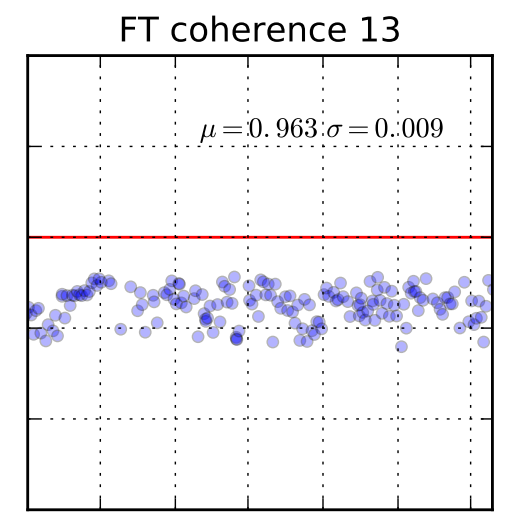

FT coherence 24

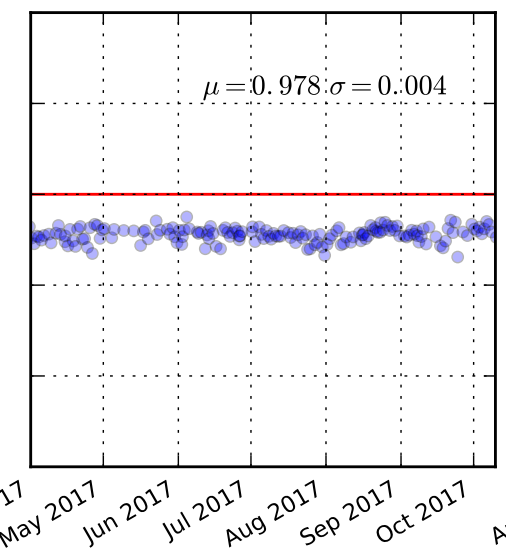

FT coherence 14

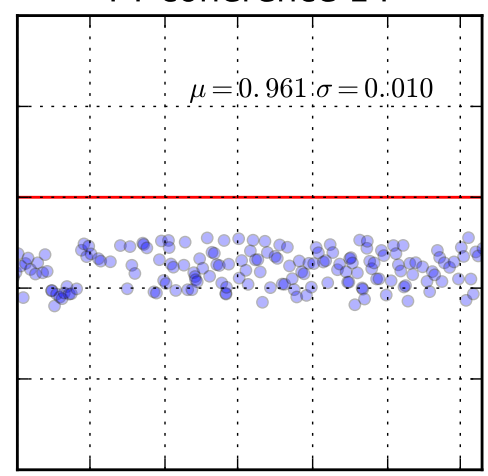

FT coherence 34

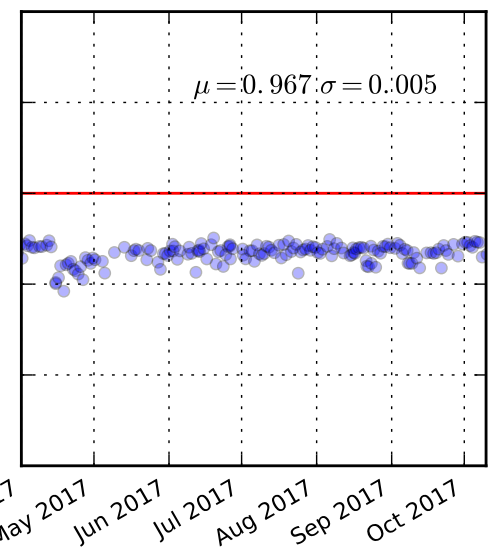

Fig. 10. Temporal variation of the FT instrumental contrast in polarized light for one polarization in medium resolution for all the baselines.

transmitted coherent flux per baseline, it should be close to $100 \%$ along the diagonal of the matrix, since it corresponds to the instrumental coherence flux, and zero elsewhere since it corresponds to the cross-talk between the baselines. We measured greater than $99 \%$ along the diagonal, and the cross-talk terms never exceeded $0.3 \%$ (Fig. 9, bottom).

Since P2VMs are part of day-time calibrations, we record them at the end of each night of observations, which allows us to follow the instrument behaviour on a day-to-day basis. All the P2VM outputs are saved as quality check parameters and can be used to check their stability over several months, as displayed in Fig. 10 for the instrumental contrast. For all the baselines, the instrumental contrast of both FT and SC combiners is above $95 \%$ and stable with an accuracy better than $1.5 \%$.

\subsubsection{Chromatic and polarization instrumental answer}

The P2VMs can be recorded with and without a Wollaston prism in the spectrometers, which allows first to calibrate the GRAVITY data in SPLIT mode (i.e. with the Wollaston prism in the spectrometers) and in COMBINED mode (i.e. without the Wollaston prism), and second to study the instrumental answer of GRAVITY with polarization. For all the quantities, the difference between the two perpendicular linear polarizations are negligible (see Fig. 9, top, for the instrumental contrast; Fig. 11 for the $\kappa$-matrix; Fig. 12 for the phase shifts; and Fig. 13 for the closure phases).

In the same way, P2VMs are computed for all the spectral channels of the spectrometers to calibrate GRAVITY data spectral channel per spectral channel, and thus they are a relevant way to study the chromatic behaviour of the quantities as the photometric throughput, the instrumental contrast, and the phase shift between the ABCD outputs. Since the couplers and the phase-shifters of the IO beam combiners are chromatic over the $K$ band, the $\kappa$-matrix coefficients are about $50 \%$ at $2.2 \mu \mathrm{m}$, but can vary by a factor of $\pm 30 \%$ from one side of the spectral band to the other (Fig. 11). With regard to the instrumental contrast, the variation over the $K$ band is of the order of $\pm 2 \%$. As far as phase is concerned, we can only discuss internal differential phases because the absolute phases of the four incident beams used to produce the P2VM are unknown. As a convention for phase reference, we assume that the phase of the A-outputs in both polarizations is null. Hence, we can discuss the differential phases of the combiner (Fig. 12). For all baselines and all the wavelengths, the $\mathrm{ABCD}$ outputs are well in quadrature (i.e. phase differences around $90^{\circ}$ ). The $\pi$-phase shifts (between the outputs $\mathrm{A}-\mathrm{C}$ and $\mathrm{B}-\mathrm{D})$ are flat at a degree-level over the spectral range since the couplers that ensure these phase shifts by design are almost achromatic. The $\pi / 2$-phase shifts (between the outputs A-D and B-C) exhibit a slope of about $\pm 7^{\circ}$ over the $K$ band due to the slight chromaticity of the phase shifters, which is in very good agreement with the simulations made by Labeye (2008).

The resulting instrumental closure phases exhibit a variation of about $\pm 30^{\circ}$ over the $K$ band (Fig. 13), which translates to a closure phase variation of $0.03^{\circ}$ per spectral channel, less than $0.3^{\circ}$ per spectral channel, and about $5^{\circ}$ per spectral channel in high, medium, and low spectral resolution, respectively.

These chromatic variations are taken into account in the P2VM and can be calibrated for all the interferometric quantities derived with the GRAVITY pipeline. The bandwidth smearing effect on all these quantities should be taken into account, besides in the low spectral resolution mode. 

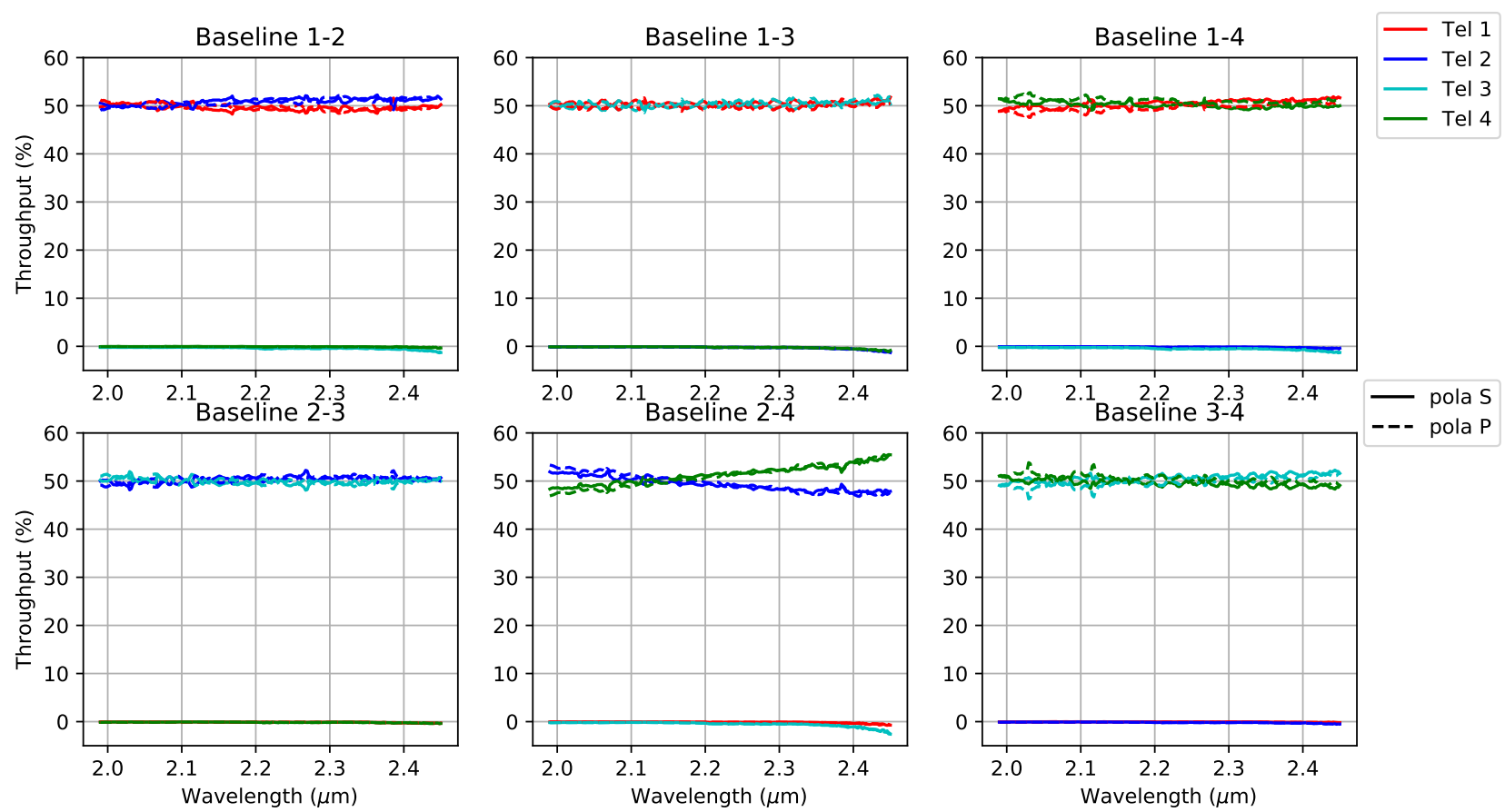

Fig. 11. Coefficients of the $\kappa$-matrix as a function of the wavelength for the two linear polarizations (solid and dashed lines, respectively).
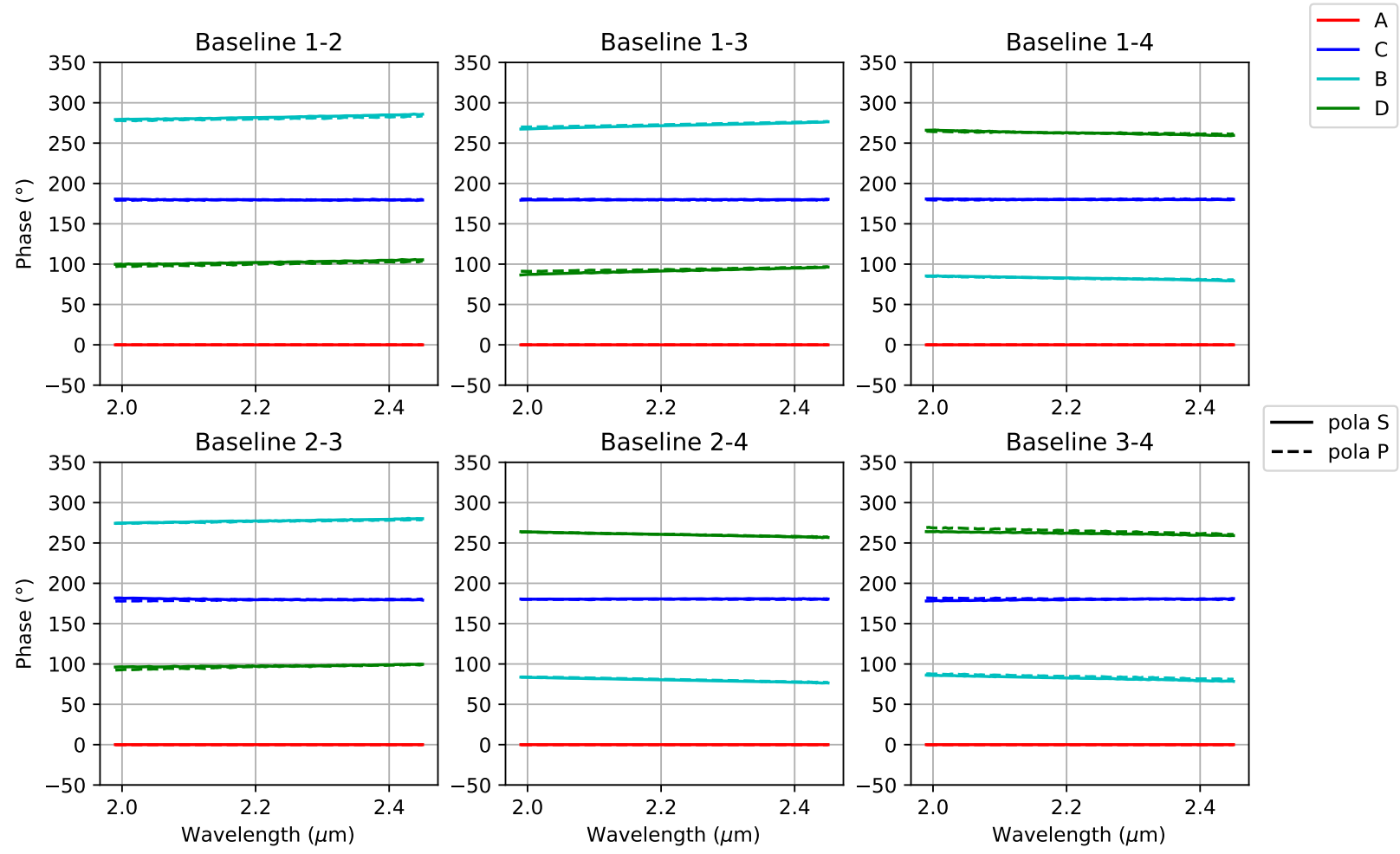

Fig. 12. ABCD phases vs. wavelength for all the baselines and the two linear polarizations (solid and dashed lines, respectively). Contrary to Fig. 6 , to allow the variation with wavelength to be seen, $\mathrm{A}$ is taken as the phase reference.

\subsubsection{Operation at $200 \mathrm{~K}$}

Finally, a main fact when operating in $K$ band is to reduce the thermal background seen by the detector as much as possible. That is why the IO beam combiners of GRAVITY have been cooled down to $200 \mathrm{~K}$. Their contribution in terms of thermal emission could only be estimated when installed in the GRAVITY cryostat.
We recorded on the SC detector in medium-resolution longexposure $(30 \mathrm{~s})$ darks, i.e. integrations without incident flux to estimate the thermal emission seen by the detector. For these recordings, as for all GRAVITY recordings, the metrology laser is back-injected at the output of the IO beam-combiners. An example of a $30 \mathrm{~s}$ dark is shown in Fig. 14. The 24 outputs are vertically aligned and spectrally dispersed along the horizontal axis. Signal above the noise level is only detected in the $1.95-2.15 \mu \mathrm{m}$ 

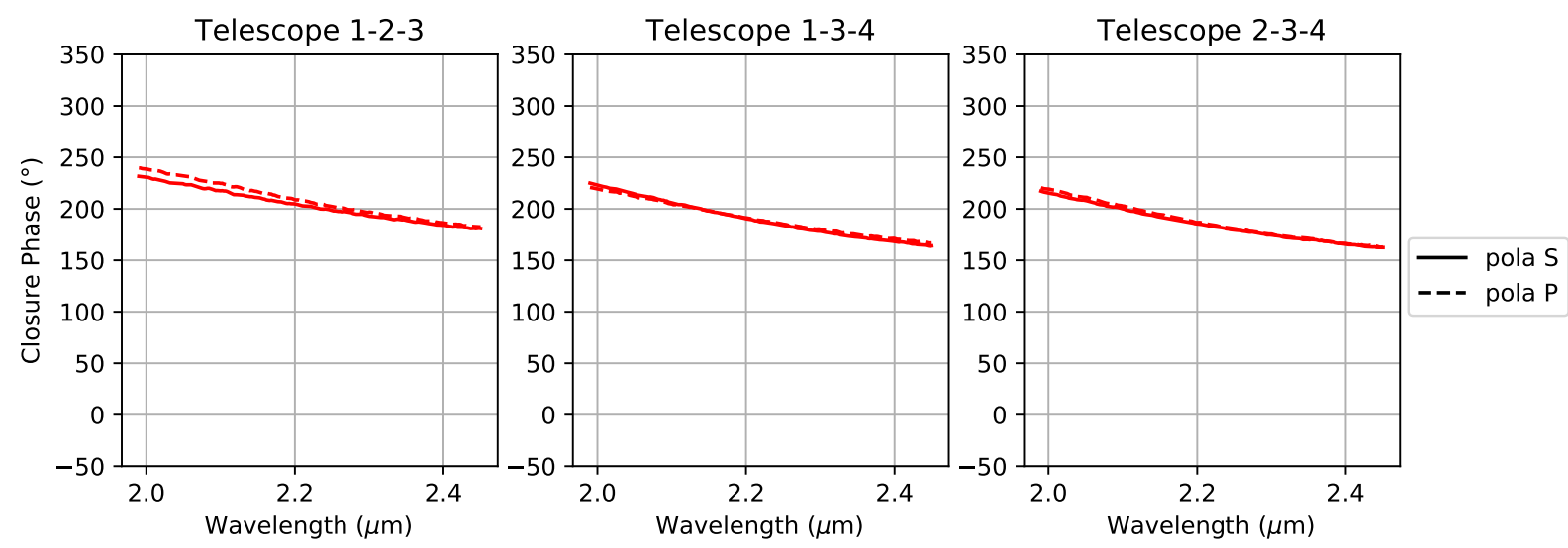

Fig. 13. Closure phases for the three independent triplets as a function of the wavelength for the two linear polarizations (solid and dashed lines, respectively).

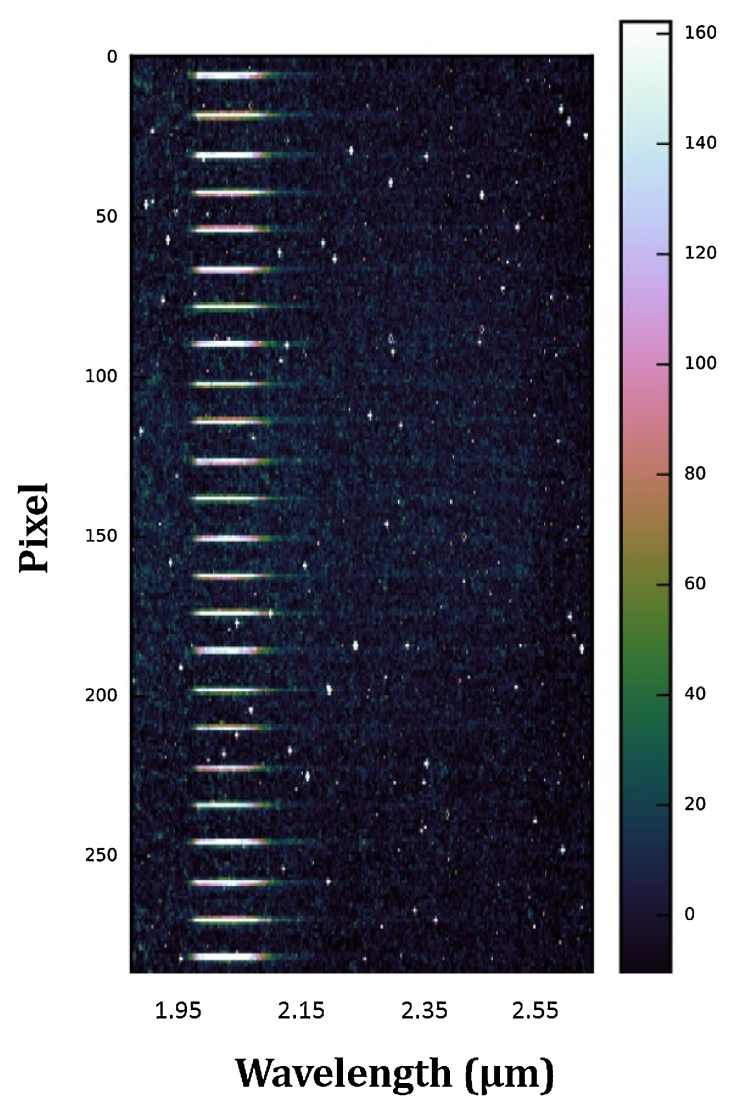

Fig. 14. Dark frame of $30 \mathrm{~s}$ recorded on the SC detector in medium spectral resolution. The colour-scale provides the counts in ADU.

range. This flux at the shortest wavelengths has been identified as fluorescence in the fluoride fibres and a Raman effect induced by the metrology laser excitation (Durteste et al. 1985). Except for the fluorescence, this dark can be directly compared with the simulation in Fig. 2; we thus validate that the IO combiners at $200 \mathrm{~K}$ do not limit the sensitivity of GRAVITY because of thermal emission at wavelengths longer than $2.15 \mu \mathrm{m}$. The minute-long coherent integrations and the limiting magnitude of $m_{K} \sim 17$ mag already obtained by the GRAVITY instrument in low spectral resolution (GRAVITY Collaboration 2017) clearly confirm this finding, and even point towards a beam combiner colder than expected, probably due to a conduction effect of the very close cold mask (Fig. 3, top).

\section{Conclusion}

Within the context of the GRAVITY instrument, we have for the first time optimized the performance of four-telescope beam combiners working in the near-infrared $K$ band. To fulfil the very demanding throughput specification of the project, we have optimized the design and the technological process for the integrated optics chip manufacturing. In addition, we have designed the whole integrated optics assembly so that it can be put inside the GRAVITY cryostat and be cooled down to $200 \mathrm{~K}$ to reduce thermal emission. The final integrated optics assemblies exhibit a global throughput over the $K$ band higher than $55 \%$ and an instrumental contrast higher than $95 \%$ in polarized light, which is well within the GRAVITY specifications. Thanks to these long-term efforts and dedicated developments, we have managed to manufacture the most complex integrated optics beam combiner in the specific $K$ spectral band and to successfully extend the maturity domain of the integrated optics technology from $H$ to $K$ band.

Acknowledgements. GRAVITY is developed in a collaboration by the Max Planck Institute for Extraterrestrial Physics, LESIA of the Paris Observatory and IPAG of Université Grenoble Alpes/CNRS, the Max Planck Institute for Astronomy, the University of Cologne, the Centro Multidisciplinar de Astrofisica Lisbon and Porto, and the European Southern Observatory. The authors thank the technical, administrative, and scientific staff of the participating institutes and at the La Silla Paranal Observatory for their extraordinary support during the development, installation, and commissioning of GRAVITY. K. Perraut and L. Jocou warmly thank P. Puget, L. Pallanca, P. Bourget, N. Schuhler, and L. Jochum for their fruitful support at different steps of the beam combiner development and integration. We thank the anonymous referee for the fruitful comments that help to improve the paper. We acknowledge the funding support of CNRS/INSU, Agence Nationale de la Recherche contract \#ANR-06-BLAN0421, LabEx OSUG@2020 (Investissements d'avenir ANR10LABX56), Action Spécifique ASHRA of CNRS/INSU and CNES, and Observatoire des Sciences de l'Univers de Grenoble(OSU). This work has been partially supported by the National Research Agency (ANR) through the French "Recherche Technologique de Base" Programme.

\section{References}

Anugu, N., Amorim, A., Gordo, P., et al. 2018, MNRAS, 476, 459

Benech, P. 1997, in Review on Integrated Optics Technology, eds. P. Kern \& F. Malbet, 115

Benisty, M., Berger, J.-P., Jocou, L., et al. 2009, A\&A, 498, 601

Berger, J. P., Haguenauer, P., Kern, P., et al. 2001, A\&A, 376, L31

Berger, J.-P., Haguenauer, P., Kern, P. Y., et al. 2003, in Interferometry for Optical Astronomy II, ed. W. A. Traub, Proc. SPIE, 4838, 1099

Coudé du Foresto, V., Perrin, G., Mariotti, J.-M., Lacasse, M., \& Traub, W. 1997, The FLUOR/IOTA Fiber Stellar Interferometer, eds. P. Kern \& F. Malbet, 115 
Deen, C., Kolb, J., Oberti, S., et al. 2016, in Adaptive Optics Systems V, Proc. SPIE, 9909, 99092M

Durteste, Y., Monerie, M., \& Lamouler, P. 1985, Electron. Lett., 21, 723

Eisenhauer, F., Perrin, G., Brandner, W., et al. 2011, The Messenger, 143, 16

GRAVITY Collaboration, Abuter, R., et al. 2017, A\&A, 602, A94

Haug, M., Haussmann, F., Kellner, S., et al. 2012, in Optical and Infrared Interferometry III, Proc. SPIE, 8445, 84452V

Kern, P., \& Malbet, F. 1997, Astrofib'96: Integrated optics for Astronomical interferometry, eds. P. Kern \& F. Malbet (Grenoble: Lab. d'Astrophysique, Obs. de Grenoble - Univ. J. Fourier/CNRS)

Labeye, P. 2008, PhD thesis, [arXiv:0904.3030]

Lacour, S., Eisenhauer, F., Gillessen, S., et al. 2014, A\&A, 567, A75

Lapeyrere, V., Kervella, P., Lacour, S., et al. 2014, in Optical and Infrared Interferometry IV, Proc. SPIE, 9146, 91462D

Laurent, E., Rousselet-Perraut, K., Benech, P., et al. 2002, A\&A, 390, 1171

Le Bouquin, J.-B. J., Berger, J.-P., Labeye, P. R., et al. 2004, in New Frontiers in Stellar Interferometry, ed. W. A. Traub, Proc. SPIE, 5491, 1362
Le Bouquin, J.-B., Labeye, P., Malbet, F., et al. 2006, A\&A, 450, 1259 Le Bouquin, J.-B., Berger, J.-P., Lazareff, B., et al. 2011, A\&A, 535, A67

Lippa, M., Gillessen, S., Blind, N., et al. 2016, in Optical and Infrared Interferometry and Imaging V, Proc. SPIE, 9907, 990722

Malbet, F., Kern, P., Schanen-Duport, I., et al. 1999, A\&AS, 138, 135

Mehrgan, L. H., Finger, G., Eisenhauer, F., \& Panduro, J. 2016, in Optical and Infrared Interferometry and Imaging V, Proc. SPIE, 9907, 99072F

Mérand, A., Abuter, R., Aller-Carpentier, E., et al. 2014, in Optical and Infrared Interferometry IV, Proc. SPIE, 9146, 91460J

Monneret, S., Huguet-Chantôme, P., \& Flory, F. 2000, J. Opt. A: Pure Appl. Opt., 2,188

Mottier, P. 1997, in Integrated Optics and Micro-optics at LETI, eds. P. Kern \& F. Malbet, 115

Scheithauer, S., Brandner, W., Deen, C., et al. 2016, in Adaptive Optics Systems V, Proc. SPIE, 9909, 99092L

Straubmeier, C., Yazici, S., Wiest, M., et al. 2014, in Optical and Infrared Interferometry IV, Proc. SPIE, 9146, 914629

Tatulli, E., Millour, F., Chelli, A., et al. 2007, A\&A, 464, 29 